\title{
Characteristics of selenium polysaccharide from sweet corncob and its effects on non-enzymatic glycosylation in vivo
}

\author{
Zhili Wang, Xin Wang ${ }^{*}$, Weiye Xiu and Yongqiang Ma
}

\begin{abstract}
Selenium polysaccharide is an organic selenium compound, which has attracted much attention because of its unique biological properties. In the current study, a new selenium polysaccharide (Se-SCP) was synthesised, and its structure and effects on non-enzymatic glycosylation in vivo were studied. The molecular weight of Se-SCP was $9.02 \mathrm{~g} / \mathrm{mol}$, and its molecule was mainly composed of Man, Gal, GluA, Ara, Glu, Fuc, Rha, GalA, and Xyl. Selenium was present both as $\mathrm{C}-\mathrm{O}-\mathrm{Se}$ and $\mathrm{Se}=\mathrm{O}$. Compared with non-selenised sweet corncob polysaccharide (SCP) treatment, the blood glucose level in mice decreased considerably after Se-SCP treatment. Additionally, it improved oral glucose tolerance (OGTT) and significantly reduced the production of advanced glycation end-products (AGEs) $(P<0.05)$, compared with the model group. In addition, Se-SCP exhibited a positive effect on the morphology of the pancreas and liver. The current study elucidated the physiological and pharmacological effects of selenium polysaccharides and provided a basis for future studies on selenium polysaccharides.
\end{abstract}

Keywords: Sweet corncob, Non-enzymatic glycosylation, Polysaccharide, Selenium, AGEs

\section{Introduction}

Type 2 diabetes mellitus (T2DM) is a prevalent metabolic disease with islet $\beta$-cell dysfunction and has organ insulin resistance as its crucial aspect. [1]. Simultaneous hyperglycaemia, insulin resistance, and lipid peroxidation often lead to cross-linked proteins. The resulting changes in protein structure render these proteins more resistant to degradation. This increases the accumulation of advanced glycation end-products (AGEs) in cells and tissues, leading to many chronic diseases, such as diabetes, renal failure, and atherosclerosis $[2,3]$.

AGEs are the end-products of a series of chemical reactions initiated by the combination of carbonyl groups in reducing sugars with free amino groups in proteins, lipids, and nucleic acids [4]. Excessive accumulation

\footnotetext{
*Correspondence: wangxinfood@163.com

Present Address: Heilongjiang Provincial Key Laboratory of Cereal and Comprehensive Processing of Cereal Resources, Harbin University
} of Commerce, Harbin 150076, China of AGEs in the body may promote oxidative stress in humans, which modifies proteins mainly through 2 pathways, oxidative phosphorylation and fatty acid oxidation. This ultimately leads to diabetes mellitus through various pathways, such as promoting protein glycosylation, binding to the receptor of advanced glycation endproducts (RAGE), and inducing oxidative stress [5]. The drugs currently marketed for diabetes treatment, including metformin, glipizide, and aspirin, do not entirely eradicate the disease; blood sugar levels can only be controlled by lifelong medication [6]. Because many Western medicines have specific toxic or adverse effects, their long-term use may cause serious bodily harm. Thus, it is necessary to identify other methods of AGE inhibition [7]. Indeed, interest has increased in identifying natural foods that can be used for diabetes treatment.

An essential element for human health, Selenium enhances the body's antioxidant capacity and resistance to related diseases; a direct relationship between selenium and human health exists [8-10]. Polysaccharides 
have various physiological functions, many of which are associated with anti-ageing and cancer prevention $[11,12]$. Inorganic selenium has cumulative toxicity and mutagenicity, whereas organic selenium has low toxicity and side effects, which can provide better play to the role of selenium [13]. Therefore, organic selenium-polysaccharide compound, generated through the combination of inorganic selenium and polysaccharides, will have the physiological and pharmacological functions of both selenium and polysaccharides. Huang discovered that the biological activity of Grifola frondosa after selenium treatment was greater than that of selenium plus G. frondosa polysaccharide, which is more easily absorbed and used by the human body [14]. Gu revealed that 2 components isolated from selenium-rich tea exhibited more substantial antioxidant effects than those in non-selenium tea. [15].

Recently, the molecular modification of polysaccharides has become increasingly sophisticated. The highlevel aspects of polysaccharide structure, such as chain flexibility and spatial conformation, are closely related to polysaccharide activity. To improve the biological activity of polysaccharides, we must investigate polysaccharide molecular modification and structural transformation [16].

Sweet corncob polysaccharide (SCP-80-I) is a watersoluble polysaccharide extracted from sweet corncob. Studies have revealed that corn silk polysaccharide exhibits a protective effect on renal function [17]. Studies have demonstrated that SCP-80-I has anti-glycosylation effects in vivo and in vitro, and this polysaccharide can inhibit glucose digestive enzymes in the small intestine [18]. The current study evaluated the structural characteristics and molecular morphology of selenium polysaccharide (Se$\mathrm{SCP}$ ) from sweet corncob using analytical techniques. Additionally, the effects of Se-SCP on non-enzymatic glycosylation were discussed. Our results revealed that the polysaccharide might have crucial pharmaceutical and health applications.

\section{Materials and methods}

\section{Materials and chemicals}

SCP-80-I was extracted as previously described [18], with some modifications. The sweet corncob was dried and crushed. Impurities were removed by passing the crushed corncob through an 80-mesh sieve. The crushed corncob was degreased by adding $\mathrm{n}$-hexane reagent in a ratio of $3: 25 \mathrm{~g} / \mathrm{mL}(\mathrm{m} / \mathrm{v})$. The residue was extracted with hot water $(1: 20 \mathrm{~g} / \mathrm{mL})$ at $100{ }^{\circ} \mathrm{C}$ for $3 \mathrm{~h}$. The supernatant was collected and concentrated at $80{ }^{\circ} \mathrm{C}$, followed by deproteinisation using the Sevag method and decolourisation using the D301R macroporous weak basic anion resin method.
Further, $80 \%$ ethanol (v/v) was added to the precipitate and incubated for $12 \mathrm{~h}$. The precipitate was freeze-dried to obtain SCP powder. SCP-80-I was prepared using cellulose column chromatography, followed by freeze-drying and Sephadex column chromatography. The cobs of KrispyKing type sweet corn were supplied by Agricultural Products Processing Co., Heilongjiang Province, China, and stored at room temperature after air drying. Streptozotocin was provided by Sigma-Aldrich. All kits were supplied by Nanjing Jiancheng Bioengineering Institute, Nanjing, China. The different organic solvents and chemicals used for the extraction were purchased from McLean, Shanghai, China.

\section{Selenium synthesis using SCP-80-I}

$\mathrm{HNO}_{3}$ solution $(0.2 \mathrm{M})$ was slowly dripped into a threenecked flask containing $N$-methylimidazole $(0.2 \mathrm{M})$. After stirring for $12 \mathrm{~h}$ in an ice bath, the solution was washed 3-5 times with ethyl acetate. Further, the ethyl acetate layer was removed and dried at $80{ }^{\circ} \mathrm{C}$ for $12 \mathrm{~h}$ to obtain viscous and transparent acidic ionic liquids (AILs).

SCP (300 mg) was added to $40 \mathrm{~mL}$ formamide, and the mixture was incubated at $80{ }^{\circ} \mathrm{C}$ for $3 \mathrm{~h}$. Further, $0.75 \mathrm{~g}$ of selenite $\left(\mathrm{H}_{2} \mathrm{SeO}_{3}\right)$ was dissolved in various AILs in an equimolar concentration at $60{ }^{\circ} \mathrm{C}$ and stirred for $2 \mathrm{~h}$. The resulting polysaccharide solution was slowly dropped into AILs and incubated at $60{ }^{\circ} \mathrm{C}$ nitrogen for $3 \mathrm{~h}$. Under isolated air, the solution was washed repeatedly with acetone until it was colourless after adding $2 \%$ ascorbic acid. After $48 \mathrm{~h}$ of dialysis, spin evaporation, precipitation, and freeze-drying were performed to obtain Se-SCP.

\section{Characterization of Se-SCP}

\section{Determination of the molecular weight of Se-SCP}

The average molecular weight (MW), number-average molecular weight (MN), and molecular weight dispersion coefficient (MW/Mn) of SCP and Se-SCP were measured using size exclusion chromatography laser light scattering analyser (SEC-MALLS: Agilient1100, Agilient lnc.,USA). The polysaccharide sample solution $(3.0 \mathrm{mg} / \mathrm{mL}, 50 \mu \mathrm{L})$ was passed through a filter with a pore size of $0.2 \mu \mathrm{m}$ and injected into the instrument. The flow rate was set at $0.5 \mathrm{~mL} / \mathrm{min}$, and measurements were performed at $25^{\circ} \mathrm{C}$. Data were collected and analysed using ASTM D3452-06 software.

\section{Determination of the monosaccharide composition of Se-SCP} We accurately weighed $10 \mathrm{mg}$ SCP and Se-SCP into a reaction kettle and added $2 \mathrm{~mL}$ trifluoroacetic acid $(4 \mathrm{~mol} / \mathrm{L})$. The mixture was heated at $100{ }^{\circ} \mathrm{C}$ for $3 \mathrm{~h}$ in an oven for hydrolysis. After cooling at room temperature, the mixture was vacuum-dried in a vacuumdrying oven at $70{ }^{\circ} \mathrm{C}$ under reduced pressure. After the 
mixture was fully hydrolysed and dried, we added $1 \mathrm{~mL}$ pyridine, followed by the immediate addition of $1 \mathrm{~mL}$ silylation reagent to prevent water in the air from entering the mixture. After thoroughly shaking the resulting mixture to dissolve all solids, the mixture was allowed to react in a constant-temperature drying oven at $50{ }^{\circ} \mathrm{C}$ for $40 \mathrm{~min}$. After cooling to room temperature, we determined the monosaccharide composition in the supernatant using a gas chromatography-mass spectrometer (Agilent 7890A, Agilient lnc., USA) with a DB-5MS $(30 \mathrm{~m} \times 0.25 \mathrm{~mm} \times 0.25 \mu \mathrm{m})$ chromatographic column. The conditions used for gas chromatography were as follows. The injection port temperature was $280{ }^{\circ} \mathrm{C}$; the interface temperature was $280{ }^{\circ} \mathrm{C}$; the carrier gas was helium. The column pressure was $73.0 \mathrm{kpa}$; the column flow was $1 \mathrm{~mL} / \mathrm{min}$, and the split ratio was $10: 1$. The temperature program was $80{ }^{\circ} \mathrm{C}$ for $3 \mathrm{~min}$, followed by an increase to $280{ }^{\circ} \mathrm{C}$ at $10^{\circ} \mathrm{C} / \mathrm{min}$ for $5 \mathrm{~min}$. The injection volume was $1 \mu \mathrm{L}$. Mass spectrometry was performed with an ion source temperature of $200{ }^{\circ} \mathrm{C}$ and an $\mathrm{M} / \mathrm{Z}$ range of $20-800$.

\section{Infrared spectrum determination of Se-SCP}

Overall, $10 \mathrm{mg}$ of SCP or Se-SCP was added in an agate mortar, mixed with dry potassium bromide, fully ground, pressed to form tablets, and scanned to obtain the infrared spectrum within the wavenumber of $4000 \sim 400 \mathrm{~cm}^{-1}$. Further, the characteristic groups of the products were analysed.

\section{Raman spectroscopy of Se-SCP}

Lyophilised Se-SCP and SCP powders were dissolved in distilled water, dripped onto a glass slide, and placed in a Raman spectrometer in the dark. The test parameters were entered using Roman analyser operation software (Omnic 8.2). The laser wavelength was $532 \mathrm{~nm}$, and the displacement scanning range was $3000-100 \mathrm{~cm}^{-1}$. The scanning map was drawn with fluorescence intensity units (intensity a.u.) along the ordinate and Raman shift along the abscissa.

\section{XPS analysis of Se-SCP}

The MgKa line was selected as the excitation source. The energy passed by the excitation source was set at $29.35 \mathrm{eV}$. The sample bin pressure was set at $2.67 \times 10^{-7}$ pa. The voltage was set at $15 \mathrm{kV}$. The power was set at $200 \mathrm{~W}$. The binding energy test accuracy was set at $0.3 \mathrm{eV}$, and the calibration material was carbon.

\section{NMR analysis of Se-SCP}

After repeated deuterium exchange with freeze-dried heavy water $\left(D_{2} O\right)$, the sample was measured in a Bruker Avance DPX-400 superconducting nuclear magnetic resonance instrument with a probe $(5 \mathrm{~mm})$ temperature of $298 \mathrm{~K}$. The sampling frequency for the 1D NMR experiment $\left({ }^{1} \mathrm{H}\right.$ NMR and ${ }^{13} \mathrm{C}$ NMR $)$ was $100 \mathrm{MHz}$. In the ${ }^{1} \mathrm{H}$ NMR experiment, 160 scans were performed; the acquisition time was $0.1803 \mathrm{~s}$, and the spectral density was $22,222 \mathrm{~Hz}$. In the ${ }^{13} \mathrm{C}$ NMR experiment, 40 scans were performed; the acquisition time was set to $0.2015 \mathrm{~s}$, and the spectral density was $5081 \mathrm{~Hz}$. All chemical shifts were expressed in PPM, and tetramethylsilane was used as the internal standard for NMR.

\section{SEM determination of Se-SCP}

A small amount of Se-SCP and SCP samples was dissolved in absolute ethanol. The particles were dispersed by ultrasonic vibration. Further, a small sample solution was taken on a copper holder, dried under an infrared lamp, and tested the samples.

\section{AFM analysis of Se-SCP}

The sample was accurately weighed, dissolved in ultrapure water, and stirred with a magnetic stirrer for more than $12 \mathrm{~h}$ to dissolve it thoroughly. Next, $5 \mu \mathrm{L}$ of the Se-SCP and SCP solutions were dripped onto the surface of a newly stripped mica sheet and air-dried at room temperature. Further, the sample on the mica sheet was scanned using an atomic force microscope (tap mode; scanning frequency, $2.65 \mathrm{~Hz}$; force $3-4 \mathrm{NN}$ ). The images were analysed using the nanoscope software provided with the AFM.

\section{Effects of Se-SCP on non-enzymatic glycosylation in vivo Establishment of the animal model}

Wistar male mice were fed adaptively for 7 days with ad libitum food and water. We randomly allocated 10 mice to the normal control group. The remaining mice were intraperitoneally injected with STZ. The model group was fed a high-fat and high-sugar diet during this period, whereas the normal control group was fed a regular diet. After the bodyweight of each mice reached $180 \pm 20 \mathrm{~g}$, STZ (55-60 mg/kg, dissolved in PBS buffer, $\mathrm{pH}$ 4.2-4.5) was injected intraperitoneally, and the mice were fed a high-sugar diet for 3 days. The fasting blood glucose of each group was measured every 3 days after fasting the mice for $3 \mathrm{~h}$. If blood glucose was greater than $11.1 \mathrm{mmol} / \mathrm{L}$ twice in a row, the T2DM mice model was considered established successfully. The diabetic mice were divided into 7 groups: the normal control group (NC), model control group (DC), positive control group (PC), polysaccharide group (SCP), high-dose Se-SCP group (Se-SCP-H), medium-dose Se-SCP group (Se-SCP$\mathrm{M})$, and low-dose Se-SCP group (Se-SCP-L).

During the feeding period, mice in the normal and model control groups were gavaged daily with 
physiological saline. The positive group was gavaged with $200 \mathrm{mg} / \mathrm{mL}$ metformin solution every day. The mice in the polysaccharide (SCP) and Se-SCP groups were gavaged with $200 \mathrm{mg} / \mathrm{kg} \mathrm{SCP}$ and various concentrations of Se-SCP (50, 100, or $200 \mathrm{mg} / \mathrm{kg}$ ) every day for 3 weeks, respectively. During that period, the diet, water intake, physical condition, and mental condition of each mouse in each group were observed daily. Body weights and blood glucose levels were measured every 7 days. After feeding for 21 days, all mice fasted for $12 \mathrm{~h}$, and blood was taken from the fundus vein for preparation. The liver, kidney, pancreas, and heart were removed from each mouse, dissected, and weighed for follow-up tests.

This study was conducted under the Principles of Laboratory Animal Care (World Health Organization Chronicle, 1985). All operations were performed under the Institutional Animal Care and Use Committee of China Medical University and in compliance with ethical principles of laboratory animal welfare.

\section{Oral glucose tolerance test (OGTT)}

OGTTs were performed as previously described [19]. The formula used to calculate AUC was as follows:

$$
\mathrm{AUC}=0.25 \times \mathrm{A}+0.5 \mathrm{~B}+0.75 \mathrm{C}+\mathrm{D}
$$

\section{Organ indices}

The hepatosomatic, renal, and pancreatic organs were removed, cleaned, and weighed. The hepatosomatic, renal, and pancreatic indices were calculated as follows:

$$
\begin{aligned}
& \text { hepatosomatic index }=\frac{\text { hepatosomatic weight }(\mathrm{g})}{\text { body weight }(\mathrm{g})}, \\
& \text { renal index }=\frac{\text { renal weight }(\mathrm{g})}{\text { body weight }(\mathrm{g})} \\
& \text { pancreas index }=\frac{\text { pancreas weight }(\mathrm{g})}{\text { body weight }(\mathrm{g})}
\end{aligned}
$$

\section{Measurement of serum lipids and oxidative stress in mice} Serum contents of 4 blood lipids (TC, TG, LDL, and $\mathrm{HDL}$ ) were measured using respective kits following the manufacturer's instructions. Indicators of oxidative stress (SOD, MDA, CAT, and GSH) were measured in the mice serum using kits following the manufacturer's instructions. The mouse plasma was centrifuged at $15{ }^{\circ} \mathrm{C}$ and $1500 \mathrm{rpm}$ for $20 \mathrm{~min}$ to separate the supernatant, kept on ice. The relevant indices were determined using the visible spectrophotometric method.

\section{Measurement of AGEs in mice}

AGEs content in mice serum was measured using ELISA as per the manufacturer's instructions. The serum was diluted 10 times, $10 \mu \mathrm{L}$ was added into each well, and subjected to ELISA using substrate TMB for colour development and OD measurement.

\section{Statistical analysis}

Origin 8.5 software was used to analyse the data and draw the graphs. Data were expressed as mean \pm standard deviation. SPSS 18.0 software was used for data analysis. Significant differences among the groups were calculated using a one-way analysis of variance (ANOVA). $P<0.05$ was considered significant.

\section{Results and discussion \\ Characterization of Se-SCP \\ Molecular weight analysis}

Compared with the natural polysaccharide, the MW of the SCP after selenisation was slightly lower, and the molecular distribution was narrow. This was due to the addition of the selenium group to the polysaccharide and the degradation of the polysaccharide chain in the acidic environment (Table 1).

\section{Monosaccharide composition}

The gas chromatograms for SCP and Se-SCP are shown in Fig. $1 \mathrm{a}$ and $\mathrm{b}$. The main chemical components of Se-SCP were total sugar and uronic acid. SCP and Se-SCP contained the same monosaccharides: Man, Gal, GluA, Ara, Glu, Fuc, Rha, GalA, and Xyl. Galacturonic acid content was higher in Se-SCP than in SCP, which may be due to heating at a higher temperature during the selenisation and the increase in temperature led to the production of galacturonic acid [20]. However, the relative abundances of each monosaccharide differed in Se-SCP and SCP. Thus, selenisation changed the relative abundance of the SCP monosaccharides but not SCP monosaccharide content.

\section{Infrared spectrum analysis}

Compared with natural polysaccharides, selenised polysaccharides had 2 characteristic absorption peaks at 726 and $1077 \mathrm{~cm}^{-1}$ (Fig. 2). The absorption peak near $1077 \mathrm{~cm}^{-1}$ was the $\mathrm{C}-\mathrm{O}-\mathrm{H}$ stretching vibration of polysaccharides

\begin{tabular}{|c|c|c|c|}
\hline Sample & $\mathrm{Mw} \times 10^{4}(\mathrm{~g} / \mathrm{mol})$ & $\mathrm{Mn} \times 10^{4}(\mathrm{~g} / \mathrm{mol})$ & $\mathrm{Mw} / \mathrm{Mn}$ \\
\hline SCP-80-I & 7.890 & 3.14 & 2.51 \\
\hline Se-SCP-80-I & 9.02 & 3.43 & 2.62 \\
\hline
\end{tabular}
with pyran rings, which suggested that Se-SCP is a pyran ring polysaccharide. The peak at $726 \mathrm{~cm}^{-1}$ was attributed

Table 1 Homogeneity and molecular weight of SCP and Se-SCP 


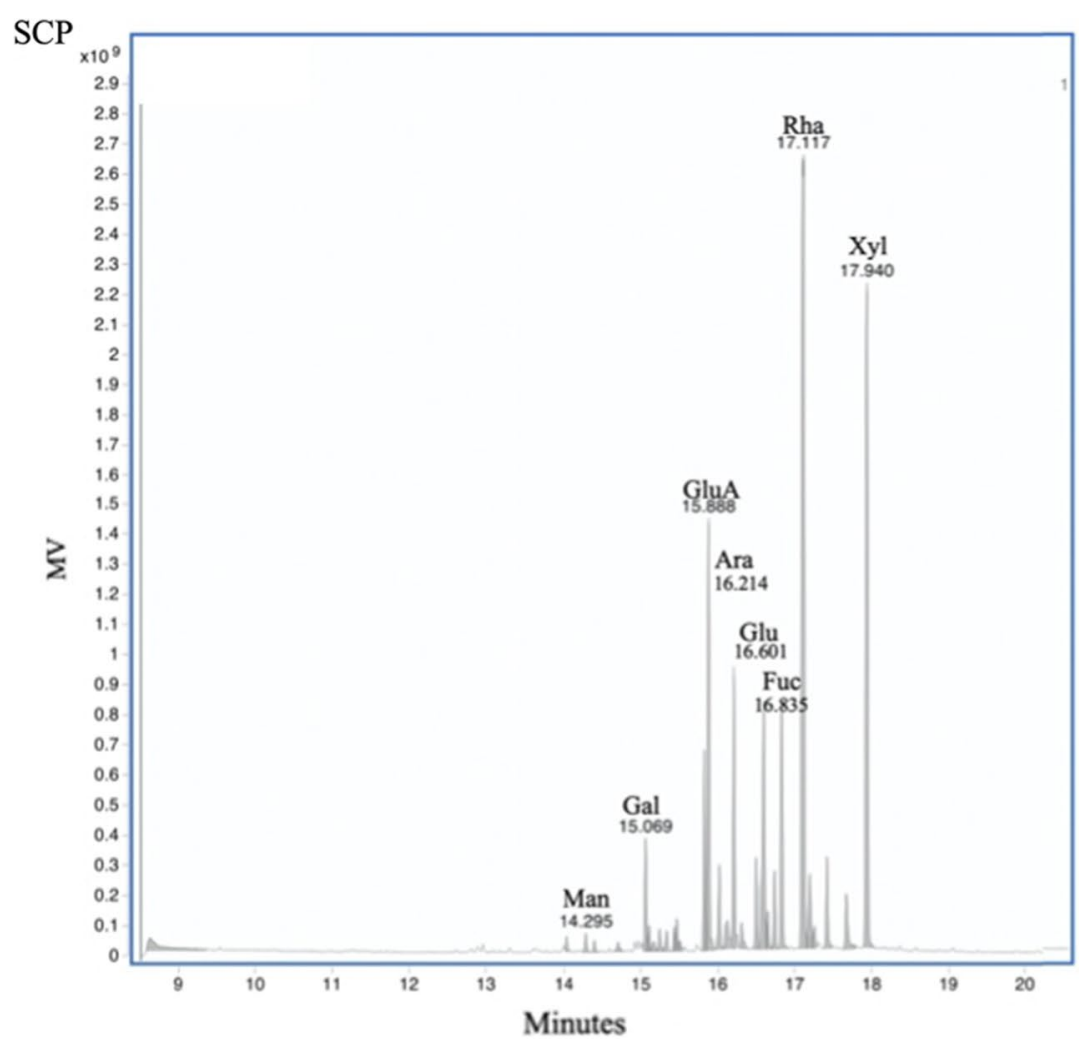

\section{$\mathrm{SeSCP}$}

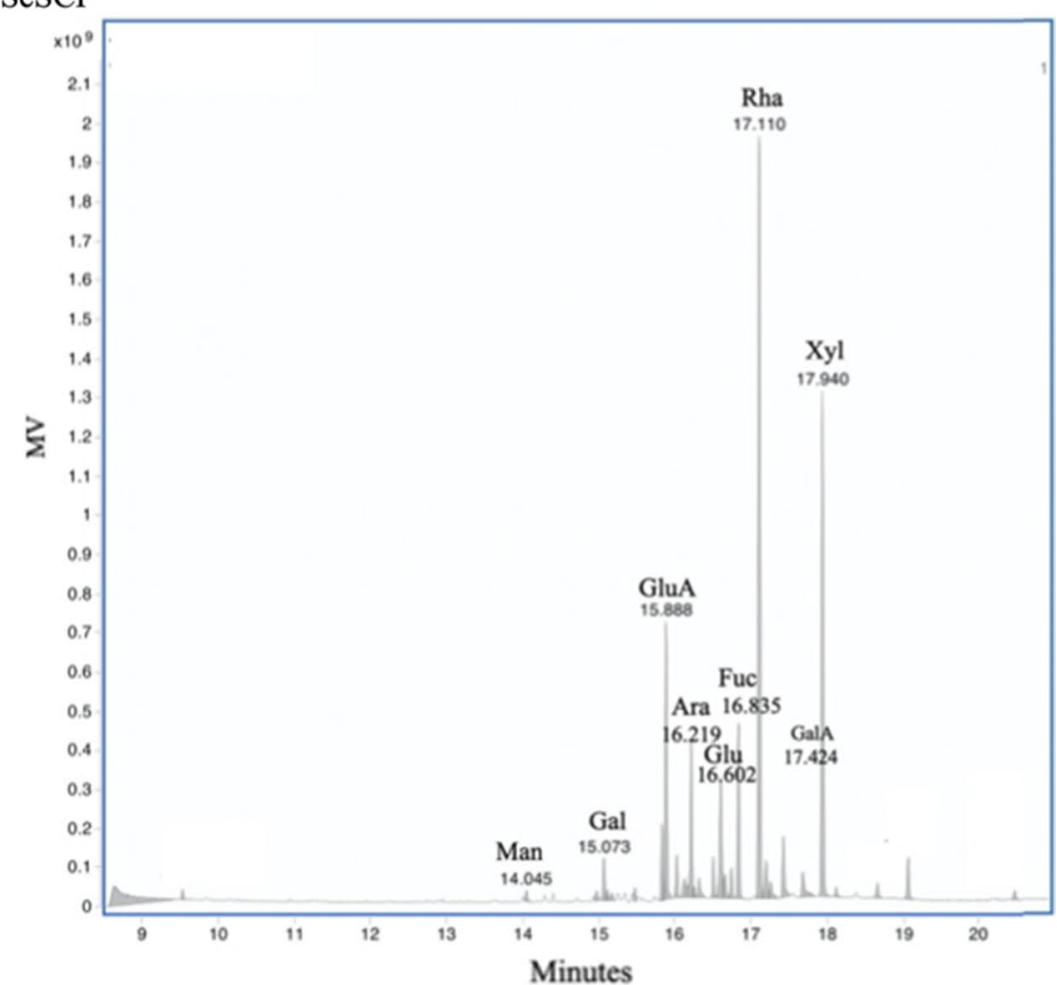

Fig. 1 GC spectra of SCP and Se-SCP 


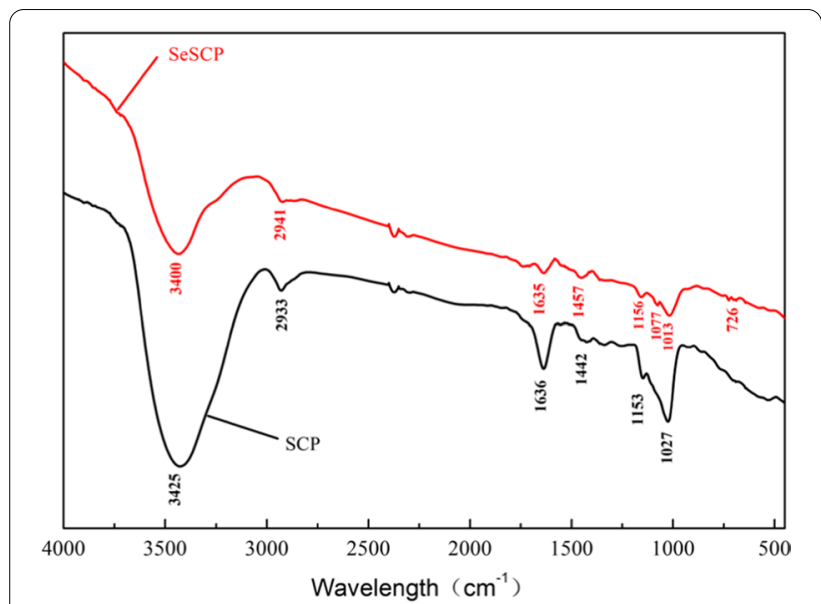

Fig. 2 Infrared spectra of SCP and Se-SCP.

to the $\mathrm{Se}=\mathrm{O}$ stretching vibration. Thus, our results indicated that selenium exists as selenite in Se-SCP because selenite forms after removing a water molecule from $-\mathrm{OH}$ in the polysaccharide molecule and $-\mathrm{OH}$ from the $\mathrm{H}_{2} \mathrm{SeO}_{3}$ molecule. This suggested that the SCP was successfully introduced into the selenium functional group. This was consistent with previous studies on pumpkin selenium polysaccharide [21] and pomegranate peel selenium polysaccharide [22].

\section{Raman spectroscopic analysis}

Raman vibration peaks for sugars are at 1120 and $1349 \mathrm{~cm}^{-1}$ [23]. The stretching vibration of the absorption peak for $\mathrm{Se}=\mathrm{O}$ in Se-SCP appeared near 732 and $861 \mathrm{~cm}^{-1}$ (Fig. 3), consistent with the characteristic absorption peak of selenate at $1077 \mathrm{~cm}^{-1}$ in the infrared spectrum of Se-SCP. These results confirmed the existence of the $\mathrm{Se}=\mathrm{O}$ bond in Se-SCP. Thus, elemental selenium reduced by selenite was successfully combined with selenium polysaccharide, changing the surface morphology of Se-SCP.

\section{NMR analysis}

To further analyse and clarify the chemical structures of the polysaccharides, SCP and Se-SCP modified by selenide were analysed using 1D NMR $\left({ }^{1} \mathrm{H}\right.$ NMR, ${ }^{13} \mathrm{C}$ NMR). Figure 4 shows the $1 \mathrm{H}$ NMR spectra of SCP and Se-SCP. Typically, the signal ranges from 5.50 to $4.90 \mathrm{ppm}$, whereas the $4.90-4.30 \mathrm{ppm}$ range is attributed to $\alpha$-heteromers and the $\beta$-heteromeric protons of heteromers [24]. Thus, our results confirmed the presence of polysaccharides in the $\alpha$ - and $\beta$-glycosidic bond skeletons. In conjunction with our monosaccharide composition analysis, the multiple $O$-methyl proton signals at 3.3-3.9 ppm confirmed that Se-SCP was composed of methoxycarbonyl $\left(-\mathrm{COOCH}_{3}\right)$. The weak signal at 1.81-2.05 ppm was attributed to the methyl proton signal of acetyl $\left(\mathrm{CH}_{2} \mathrm{CO}-\right)$, which indicated that some carboxyl groups in Se-SCP were replaced by acetyl groups. In addition, the signal at $1.09-1.23 \mathrm{ppm}$ confirmed the presence of $\beta$-1-rhap residues [25-28]. Figure 4 shows the 13C NMR spectra of SCP and Se-SCP. The peaks in the $50-110 \mathrm{ppm}$ region of SCP were examined. The resonance near the $90-110 \mathrm{ppm}$ region was attributed to galactopyranosyl (Glap, $97.68 \mathrm{ppm}$ ) and mannopyranosyl (Manp, 99.61 ppm) [29].
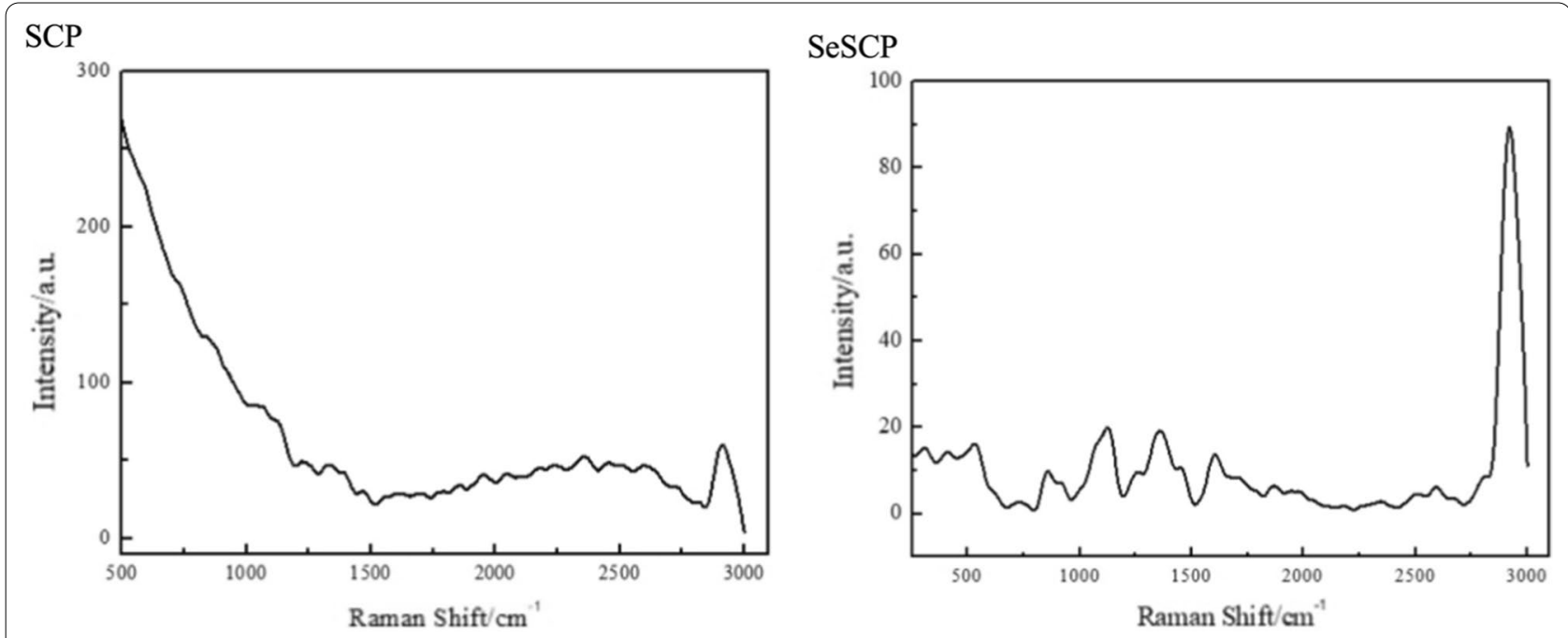

Fig. 3 Raman spectra of SCP and Se-SCP 

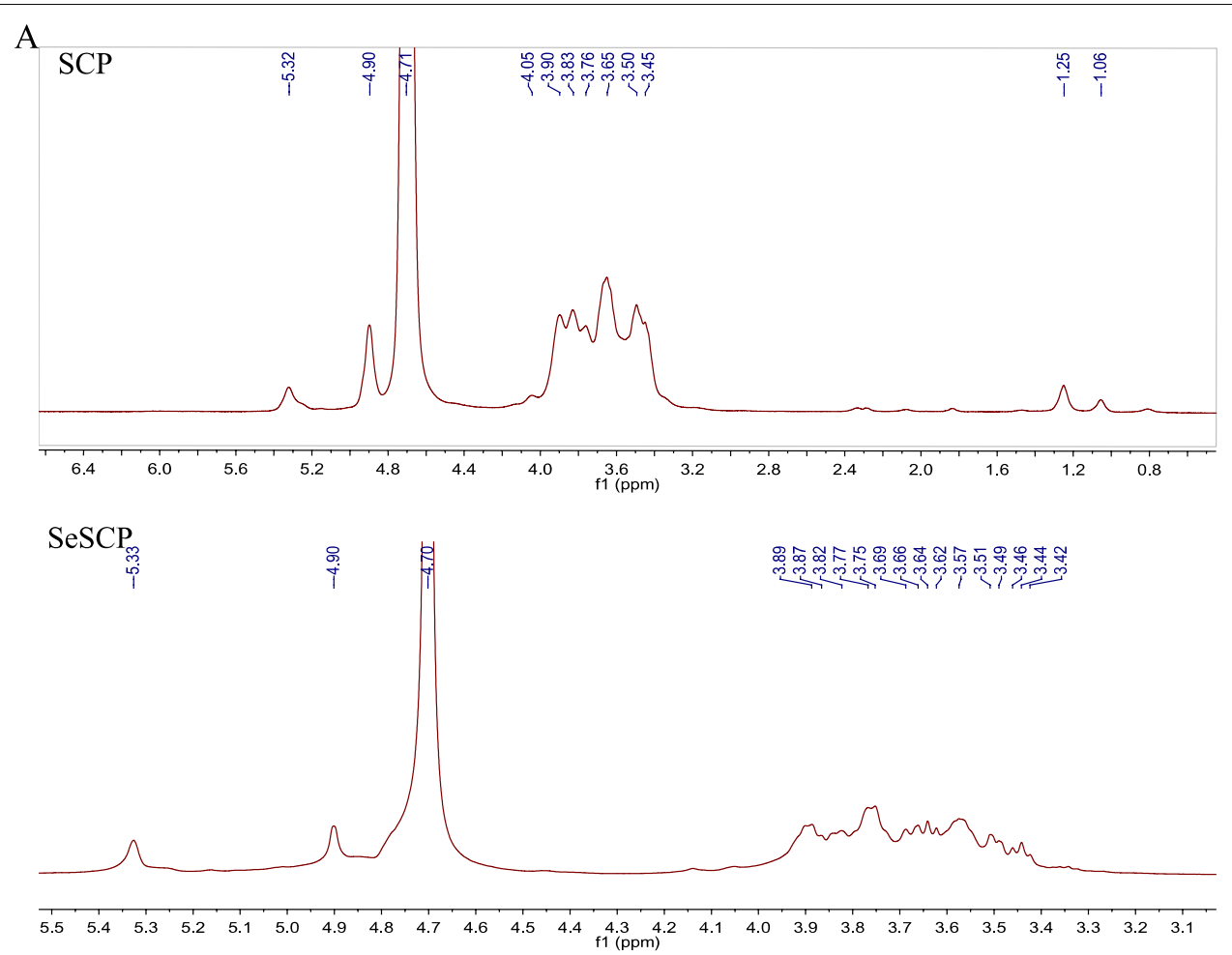

B SCP
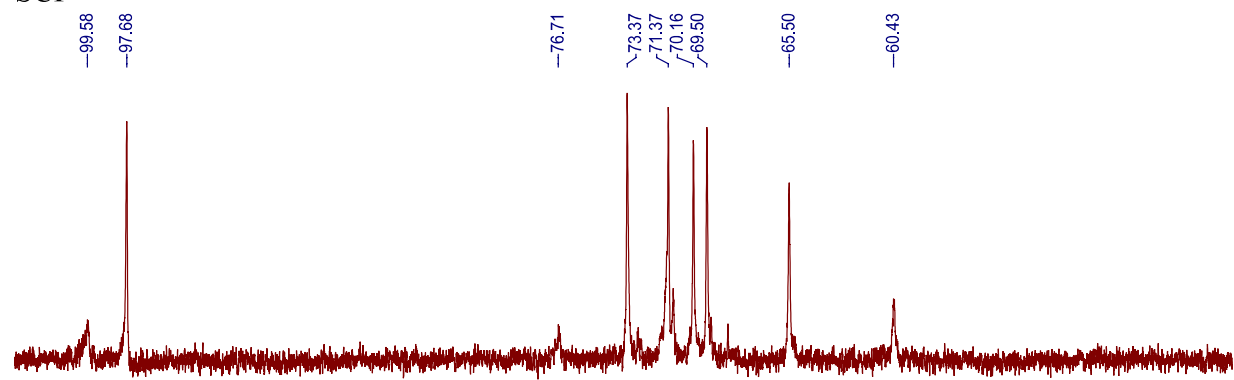
\begin{tabular}{lllllllllllllllllllllllllllllllllllll}
\hline 102 & 98 & 96 & 94 & 92 & 90 & 88 & 86 & 84 & 82 & 80 & 78 & 76 & 74 & 72 & 70 & 68 & 66 & 64 & 62 & 60 & 58 & 56 & 54 & 52 & 50 & 48 & 46 & 4
\end{tabular}
SeSCP
is

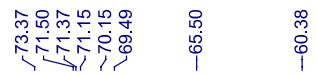

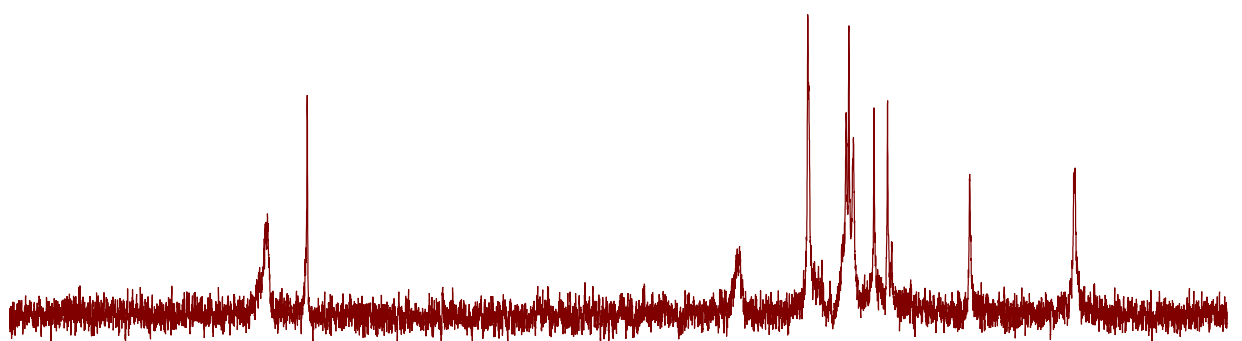

$\begin{array}{llllllllllllllllllllllllllllllllllll}110 & 106 & 102 & 98 & 96 & 94 & 92 & 90 & 88 & 86 & 84 & 82 & 80 & 78 & 76 & 74 & 72 & 70 & 68 & 66 & 64 & 62 & 60 & 58 & 56 & 54\end{array}$

Fig. $4{ }^{1} \mathrm{H}$ and ${ }^{13} \mathrm{C}$ spectra of SCP and Se-SCP 


\section{XPS analysis}

Figure 5 shows the full XPS spectra of SCP and Se-SCP. The binding energies of 284.7 and $532.3 \mathrm{eV}$ in SCP corresponded to the inner electron binding energies of $\mathrm{C}$ and $\mathrm{O}, \mathrm{C} 1 \mathrm{~s}$ and $\mathrm{O} 1 \mathrm{~s}$, respectively. The Se-SCP spectrum revealed that the binding energies of Se-SCP were 284.8 and $532 \mathrm{eV}$, corresponding to the inner electron binding energies of $\mathrm{C}$ and $\mathrm{O}$, respectively. Compared with SCP, the Se 3D peak appeared at $58.2 \mathrm{eV}$ in Se-SCP, consistent with the selenisation of SCP. Consistent with these results, previous studies had observed the Se 3D peak when the binding energy was close to $59 \mathrm{eV}$, indicating that selenium was introduced into the SCP structure as $\mathrm{Se}^{4+}$.

We observed 3 forms of $\mathrm{C}$ 1s in SCP: C-H $(284.7 \mathrm{eV})$, $\mathrm{C}-\mathrm{OH}(285.8 \mathrm{eV})$, and $\mathrm{C}=\mathrm{O}(288.2 \mathrm{eV})$. After the selenisation of SCP, binding energies between Se-SCP and C $1 \mathrm{~s}$ in SCP were compared. We observed that the binding energy was reduced because the introduction of the selenite group affected the density of the $\mathrm{C}$ electron cloud, and the oxygen content increased after selenisation. This demonstrated that selenium was successfully introduced into the SCP structure as selenite $\left(\mathrm{SeO}_{3}{ }^{2-}\right)$.

\section{SEM analysis}

SEM images of SCP and Se-SCP are shown in Fig. 6, divided into 3 multiples of $50 \mathrm{~K}, 20 \mathrm{~K}$, and $10 \mathrm{~K}$ from left to right. These images revealed that the surfaces of SCP and Se-SCP differed in both size and hardness. The surface of SCP was rough, with irregular loose voids, uneven surfaces, and particle attachment, whereas the microstructure of the Se-SCP surface was mostly irregular bands, filaments, and lamellae, with a soft surface and loose holes.

\section{AFM analysis}

Figure 7 shows $2 \mathrm{D}$ and $3 \mathrm{D}$ atomic force micrographs of SCP. Comparative observations revealed that SeSCP was structured in stacked sheets with sharp edges and corners. This was consistent with our SEM results.
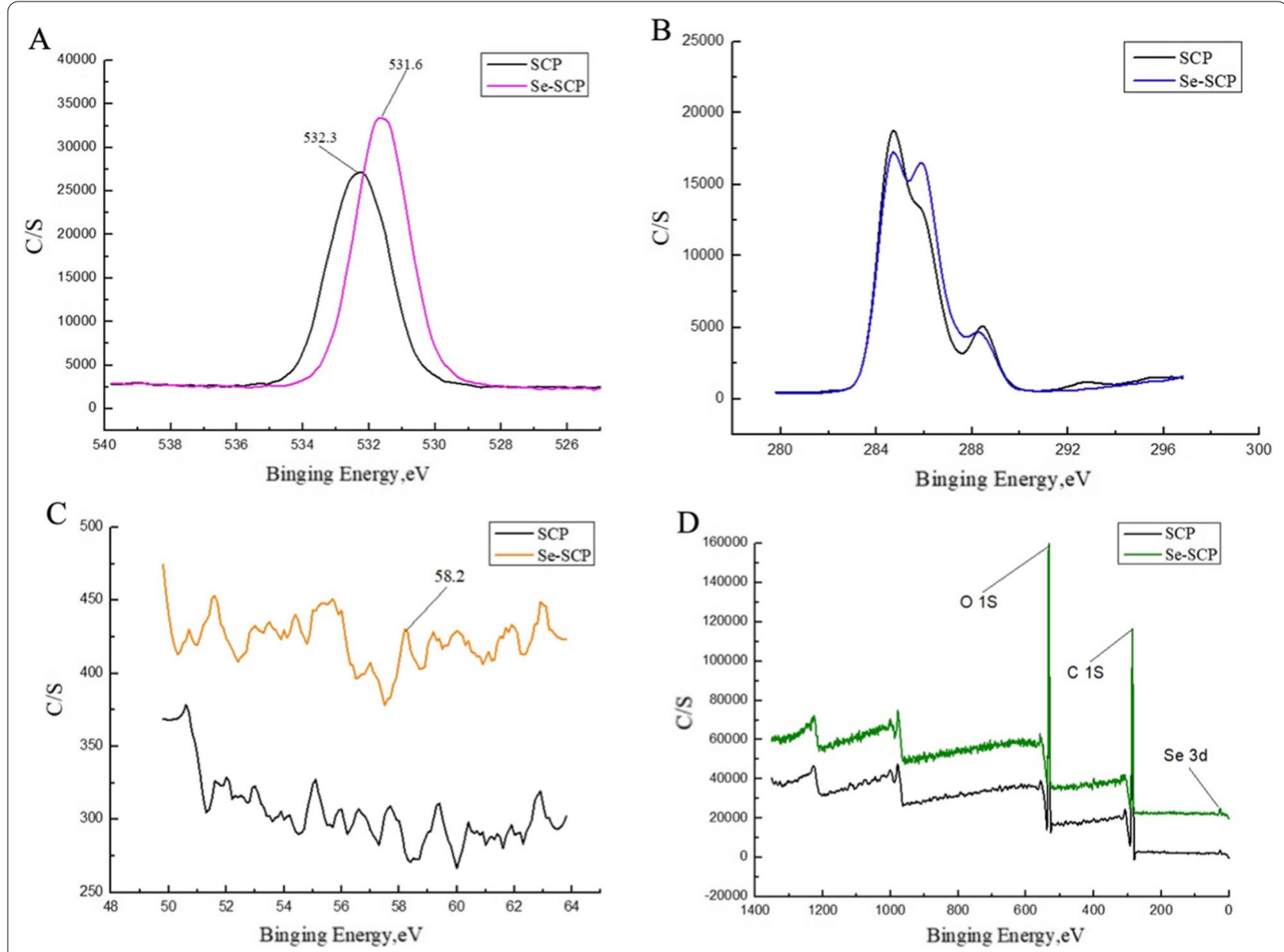

Fig. 5 XPS of SCP and Se-SCP 


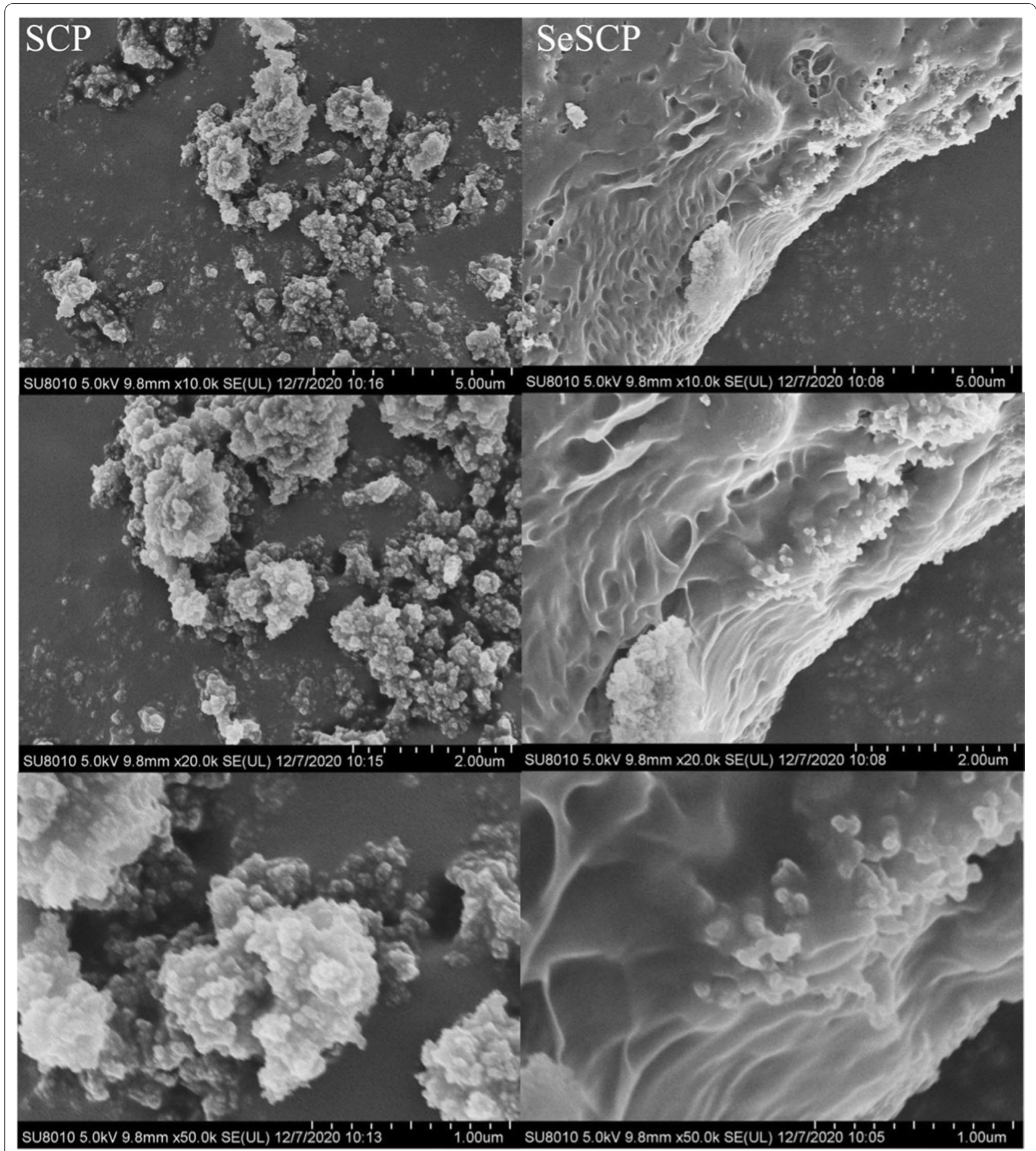

Fig. 6 SEM of SCP and Se-SCP 

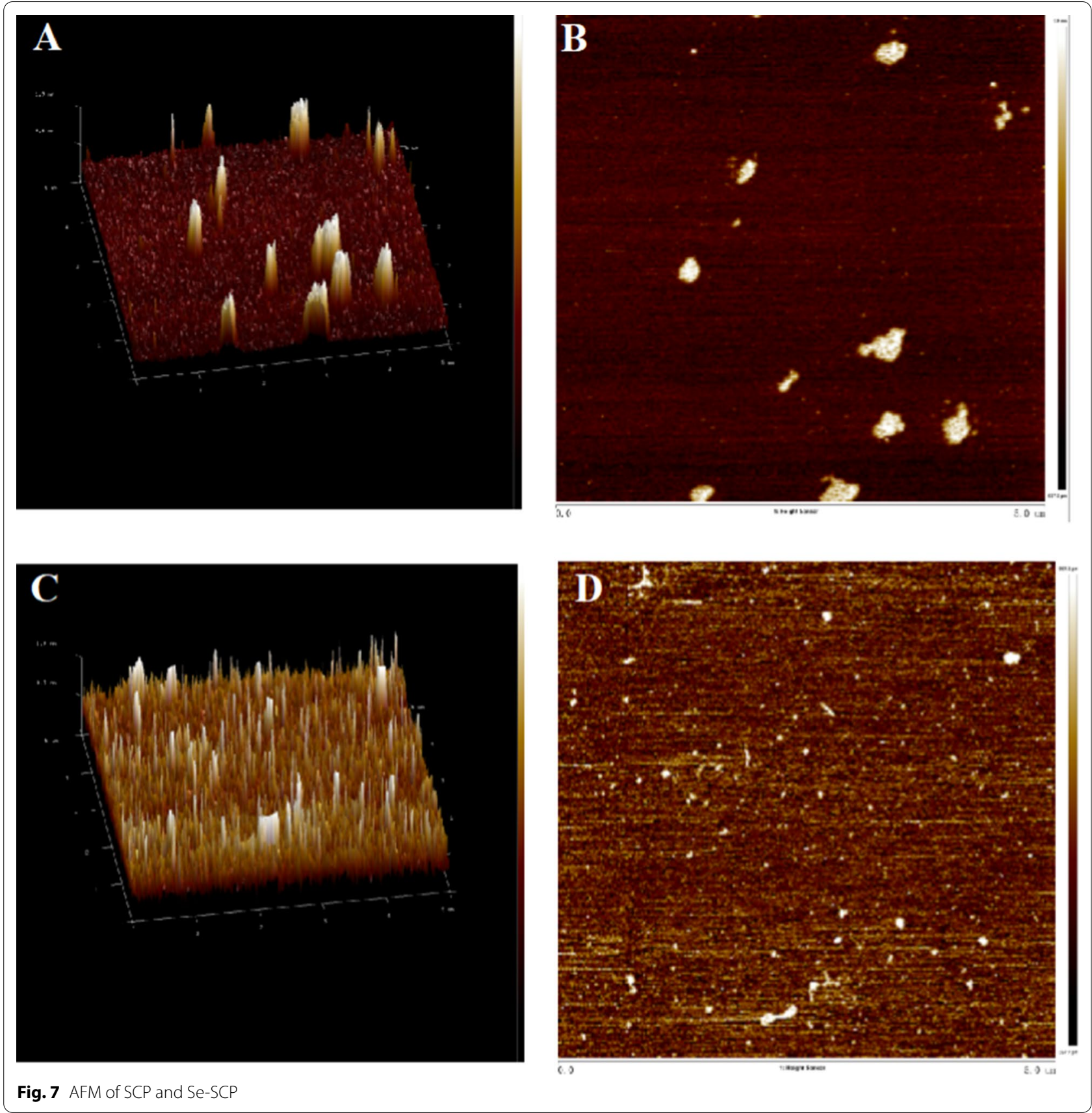

Fig. 7 AFM of SCP and Se-SCP

Se-SCP dispersed uniformly in an aqueous solution, and its spherical structure had a length of 30-150 nm and a height of 0.3-1.0 nm. Spherical structures of various sizes were formed by winding molecular chains and branched chains.
Effects of Se-SCP on non-enzymatic glycosylation in vivo Mice weight and blood glucose

The mice in the NC group were healthy and proliferated, with gradually increased body weights (Fig. 8A). In contrast, the mice's body weights in the $\mathrm{DC}, \mathrm{PC}$, 

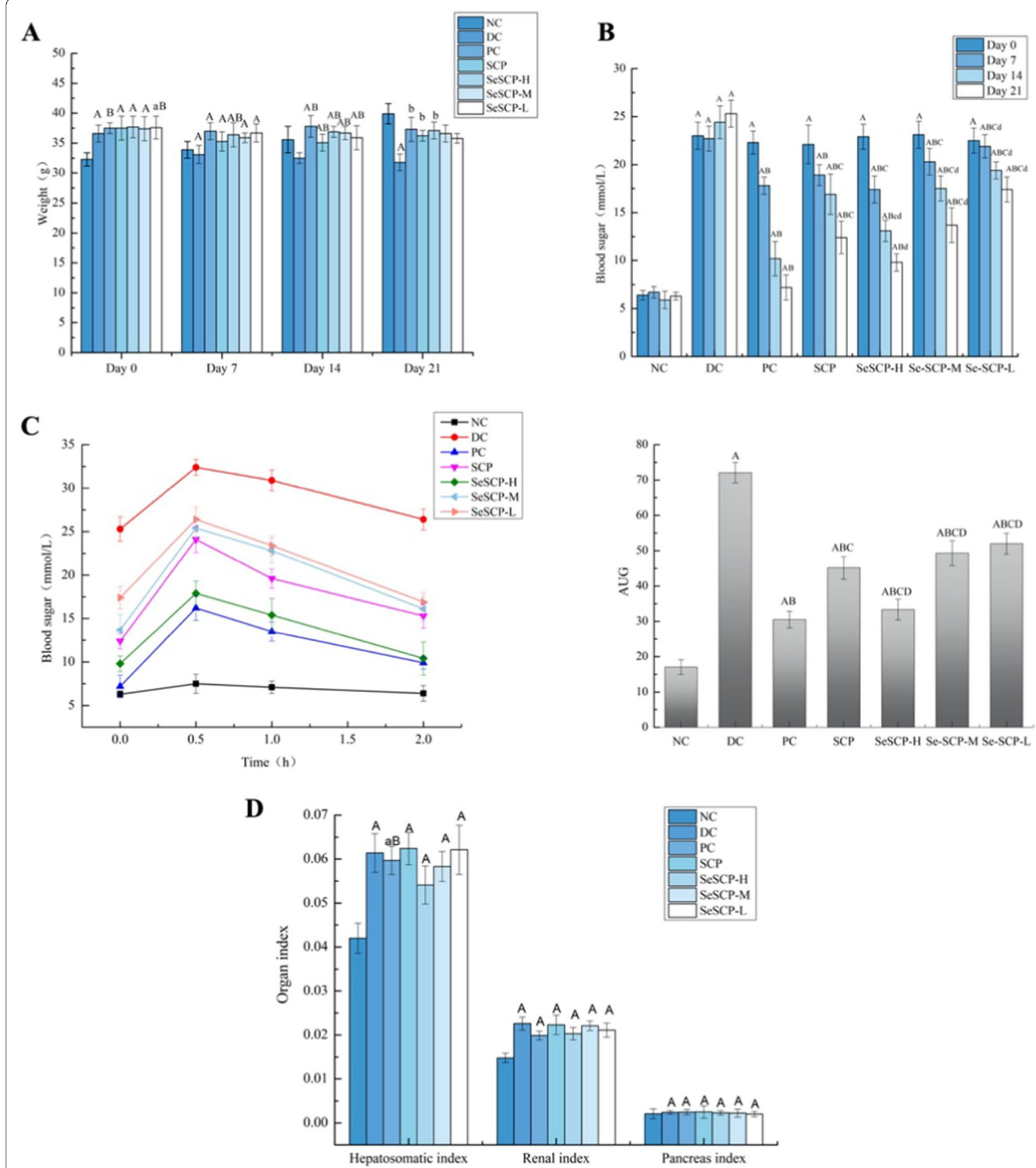

Fig. 8 Determination of indices in animal experiments (Effect of polysaccharide on A: body weight, B: blood sugar, C: AUC, D: organ indices). Compared with the NC group, a: $p<0.05$ and A: $p<0.01$; DC group, $b: p<0.05$ and $B: p<0.01 ; P C$ group: $c: p<0.05$ and $C: p<0.01 ;$ SCP group, d: $p<0.05$ and $D: p<0.01$ 
Se-SCP-H, Se-SCP-M, and Se-SCP-L groups decreased by varying amounts. During the experiment, we observed that mice in the NC group had brightly coloured fur, a good mental state, and normal agility, whereas mice in the DC group mice exhibited varying degrees of mental depression. After 21 days of gavage treatment, the fur colour and mental state of the SeSCP and PC groups slightly improved (Fig. 8B). Therefore, it can be stated that weight loss and elevated blood glucose in mice caused by diabetes could be effectively alleviated by seleno-polysaccharide treatment.

\section{Mice glucose tolerance and area under the curve}

The results of the OGTT correspond to glucose tolerance, and the ability to regulate blood glucose after administration corresponds to the level of glucose tolerance (Fig. 8C). The blood glucose curve in the NC group tended to be gentle, and blood glucose levels in the DC group were higher than those in the $\mathrm{NC}$ group at every time point. After treatment with metformin, blood glucose levels in the SCP, Se-SCP-H, Se-SCP-M, and
Se-SCP-L groups decreased significantly at each time point $(\mathrm{P}<0.01)$.

\section{Mice organ indices}

Figure 8D shows the effects of SCP and Se-SCP on the organ coefficients of T2DM mice, mainly including the hepatosomatic, renal, and pancreatic indices. These indices of T2DM mice were increased to different degrees compared with the normal group $(\mathrm{p}<0.05)$, indicating that the high-fat-fed diabetic mice revealed significant swelling of hepatosomatic, renal, and pancreas. The PC group and each dose group of SCP and Se-SCP had a certain degree of reduction in organ swelling after the respective treatment.

\section{Mice serum lipid contents}

T2DM pathogenesis is closely related to blood lipid level. Decreases in blood lipid levels help improve T2DM by reducing the production of non-enzymatic glycosylation end-products. Levels of serum TC, TG, and LDL in diabetic mice increased significantly compared with

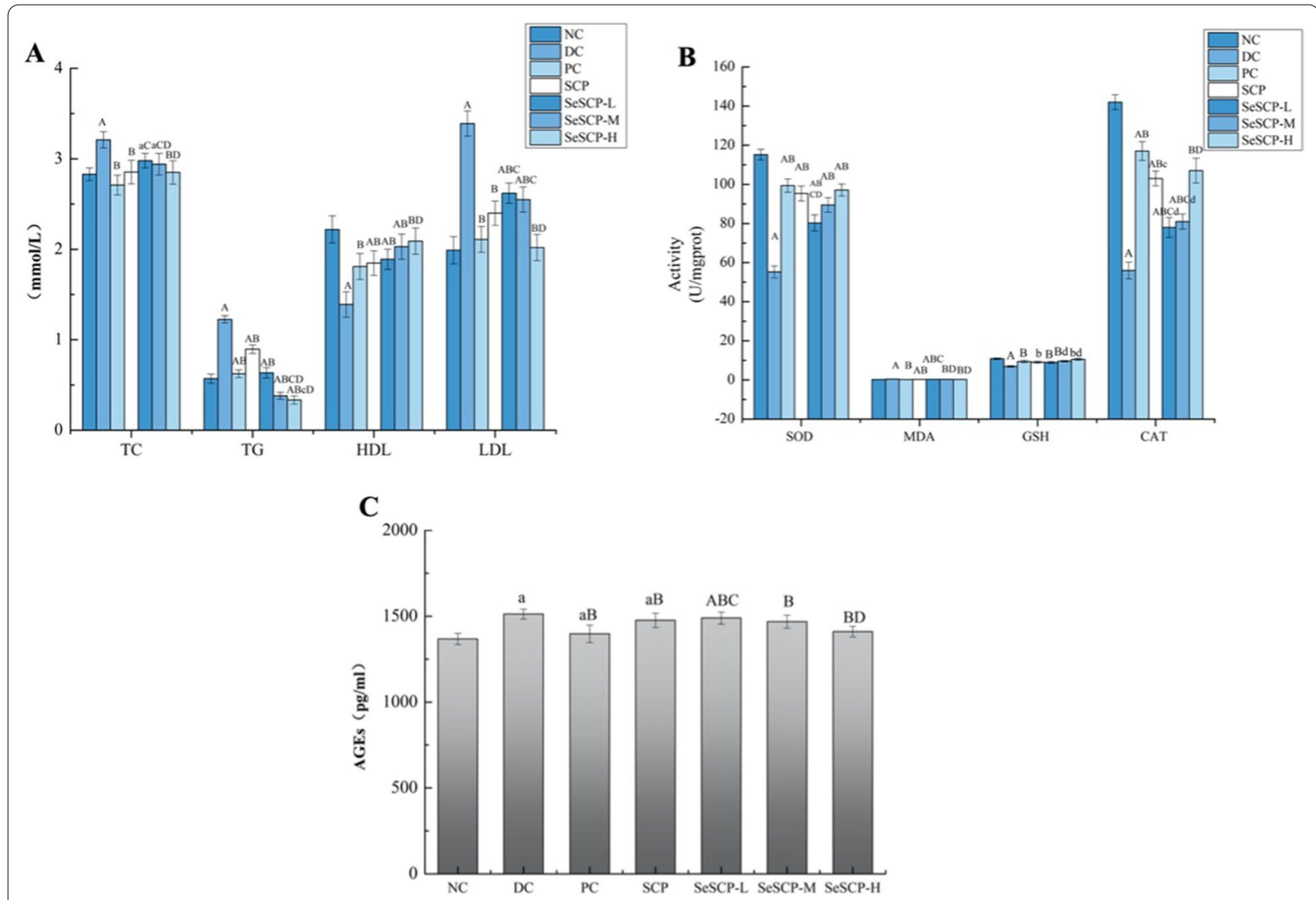

Fig. 9 Determination of indices in animal experiments (Effect of polysaccharide on A: oxidative stress, B: 4 blood lipid, C: AGEs). Compared with the NC group, a: $p<0.05$ and $A: p<0.01$; DC group, $b: p<0.05$ and $B: p<0.01 ; P C$ group: $c: p<0.05$ and $C: p<0.01 ;$ SCP group, : $p<0.05$ and $D: p<0.01$ 
the NC group, whereas HDL content decreased significantly (Fig. 9A). After 21 days of selenium polysaccharide administration, serum TC, TG, and LDL contents decreased significantly $(\mathrm{P}<0.05)$, and serum HDL content increased significantly compared with the DC group. Compared with the positive group, the metabolic effects of serum TG, HDL and LDL in the Se-SCP-H group were better than those in the positive group to some extent. This suggested that seleno-polysaccharides could inhibit glucose levels in the blood through a pathway that reduces lipid peroxidation and ultimately inhibits the non-enzymatic glycosylation process.

\section{Oxidative stress levels}

The oxidative stress response induced by AGEs is mainly mediated by AGEs and the AGE receptor axis. AGEs and their receptors mediate a series of complex signal transduction pathways in the cell, inducing the oxidative stress response, which disrupts tissues and organs' functions. The antioxidant capacity of the diabetic mice decreased significantly compared with the NC group (Fig. 9B). Specifically, MDA content in the pancreatic tissue increased significantly, whereas the activity levels of the antioxidant enzymes (SOD, GSH, and CAT) were significantly reduced. After 21-day intragastric administration of selenium polysaccharide, MDA content in the pancreas decreased, and antioxidant enzyme activity increased significantly in the diabetic mice. This suggested that seleno-polysaccharide may inhibit the non-enzymatic glycosylation process by inhibiting oxidative stress, and the effect was better than that of the SCP group. Additionally, it could be concluded from the above results that Se-SCP after selenisation had the dual antioxidant activities of polysaccharide and selenium.

\section{Effects of Se-SCP on fluorescent AGEs in mice serum}

The fluorescence intensity of the AGEs in the serum samples from mice in the DC group was significantly stronger than that in the serum samples of any other group (Fig. 9C). The fluorescence intensity of the PC group was significantly higher than that of the NC group, and that in the Se-SCP-H group was significantly lower than that in the DC group. Selenium polysaccharide inhibited AGE formation to some extent, and no significant difference among positive drug treatment levels was observed. Therefore, seleno-polysaccharides may inhibit the production of AGEs by binding to carbonyl compounds.

\section{HE staining of the liver and pancreas}

At the end of week 4 of the experimentation, HE staining revealed inflammation and necrosis in the liver tissues of diabetic mice in the DC group (Fig. 10). There was less inflammation in the positive group than in the DC group. The HE results revealed that as Se-SCP dosage increased, cell necrosis improved and inflammation decreased.

The islet structure was clear in the NC group, and the pancreatic cells were morphologically normal, evenly distributed, and well coloured. Compared with the NC group, the pancreatic cells in the DC group were disordered with more vacuoles; the nuclei were not coloured; a few nuclei were pyknotic, and islets were rare and smaller when present. In the selenium polysaccharide group, after 3 weeks of treatment, the number of pancreatic cells increased; the islet structure was clear; the boundary was clear; the cytoplasm was uniform; there were no vacuoles, and the nucleus was clear. Similar changes were observed in the PC group. This indicated that SCP after selenisation alleviated the destruction of the pancreas and islet cells caused by diabetes, and the effect was better than that in the unselenised SCP group. 

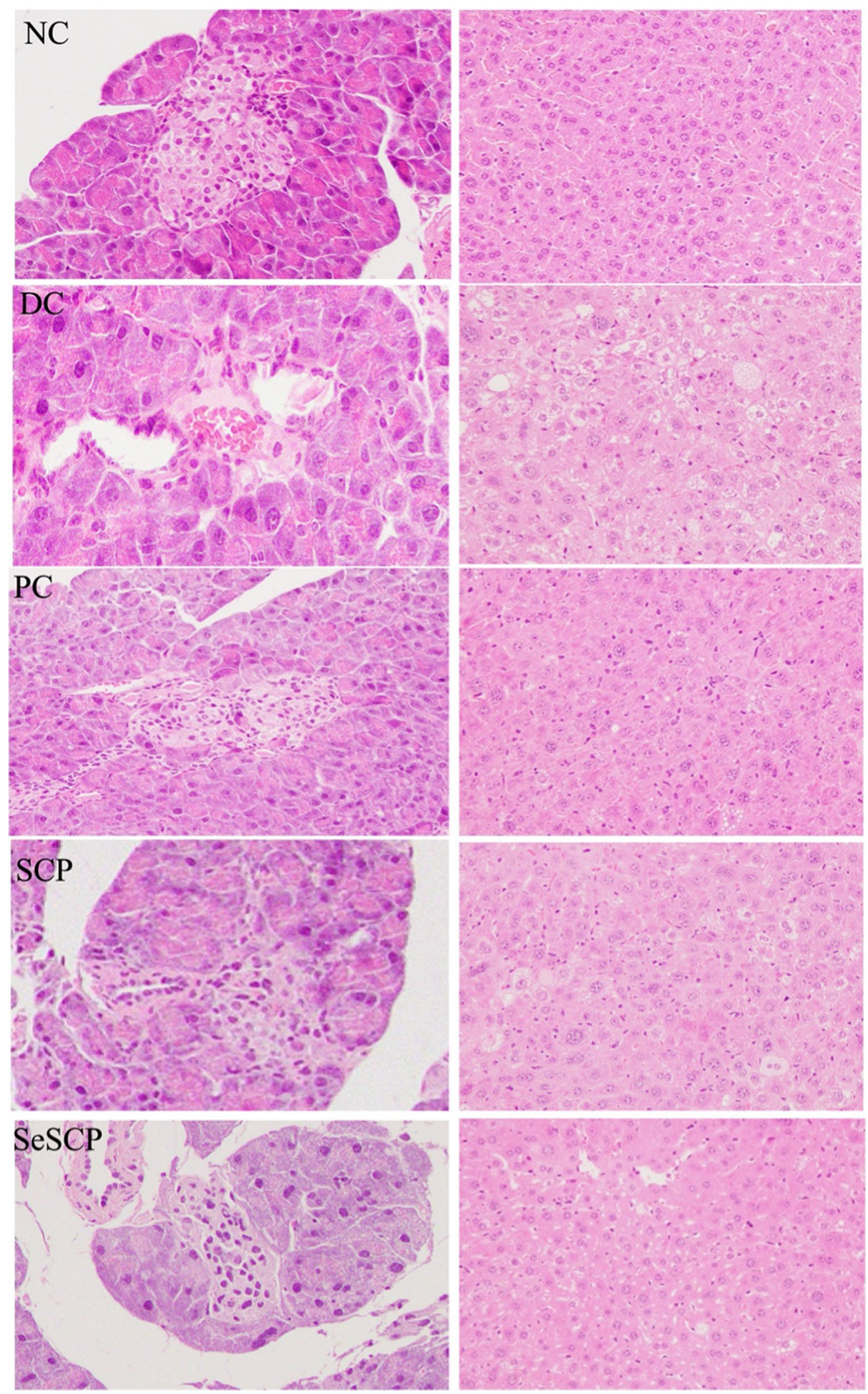

Fig. $10 \mathrm{HE}$ stained sections of the pancreas and liver 


\section{Acknowledgements}

We thank LetPub (www.letpub.com) for its linguistic assistance during the preparation of this manuscript.

\section{Authors' contributions}

Conceptualization, YM; Data curation, ZW; Formal analysis, ZW; Funding acquisition, XW and ZW; Methodology, XW; Supervision, XW; Writing-original draft, ZW; Writing - review \& editing, ZW. All authors read and approved the final manuscript.

\section{Funding}

This research was financially supported by the "Young reserve talents" special program from Harbin University of Commerce (2019CX31), Doctoral Scientific Research Start-up foundation from Harbin University of Commerce (2019DS098), Graduate innovation fund project of Harbin University of Commerce (YJSCX2019-615HSD). The authors are grateful to supports. We would like to thank LetPub (www.letpub.com.cn) for editing and proofreading this manuscript.

\section{Availability of data and materials}

The data presented in this paper are available on request from the first author and corresponding author.

\section{Declarations}

\section{Competing interests}

The authors declare no competing interests.

Received: 19 October 2021 Accepted: 14 January 2022

Published online: 07 February 2022

\section{References}

1. Oh KK, Adnan M, Cho DH (2021) Active ingredients and mechanisms of Phellinus linteus (grown on Rosa multiflora) for alleviation of Type 2 diabetes mellitus through network pharmacology. Gene 768:901-906

2. Alok R, Jamal A, Khursheed A (2018) Preferential recognition of advanced glycation end products by serum antibodies and low-grade systemic inflammation in diabetes mellitus and its complications. Int J Biol Macromol 118(B):1884-1891

3. Liu D, Ji L, Zhang D, Tong X, Pan B, Liu P, Zhang Y, Huang Y, Su J, Belinda W, Zheng $L$ (2012) Non-enzymatic glycation of high-density lipoprotein impairs its anti-inflammatory effects in innate immunity. Diabetes/Metab Res Rev 28(2):186-195

4. Peng X, Ma J, Chen F (2011) Naturally occurring inhibitors against the formation of advanced glycation end-products. Food Funct 2(6):289-301

5. Velichkova S, Foubert K, Pieters L (2021) Correction: natural products as a source of inspiration for novel inhibitors of advanced glycation endproducts (AGEs) formation. Planta Med 87:780-802

6. Soleimani AS, Amiri I, Samzadeh-kermani A, Abbasalipourkabir R, Gholamigeravand B, Shahidi S (2021) Chitosan-coated Selenium nanoparticles enhance the efficiency of stem cells in the neuroprotection of streptozotocin-induced neurotoxicity in male rats. Int J Bio Cell Bio. 141:106089

7. Yang D, Hu C, Wang X, Shi G, Li Y, Fei Y, Song Y, Zhao X (2021) Microbes: a potential tool for selenium biofortification. Metallomics 13(10):54

8. Jia Y, Zhang L, Liu X, Zhang S, Dai J, Huang J, Chen J, Wang Y, Zhou J, Zeng $Z$ (2021) Selenium can regulate the differentiation and immune function of human dendritic cells. Biometals 2:44

9. RamírezAlarcón K, Victoriano M, Mardones L, Villagran M, AlHarrasi A, AlRawahi A, CruzMartins N, SharifiRad J, Martorell M (2021) Phytochemicals as potential epidrugs in type 2 diabetes mellitus. Front Endocrinol 12:656978

10. Wei J, Wang Q, Zheng H, Wei F (2018) Research progress on non-drug treatment for blood glucose control of type 2 diabetes mellitus. Chin J Integra Med 24(10):723-727

11. Zhang S, Zhang H, Shi L, Li Y, Tuerhong M, Abudukeremu M, Cui J, Li Y, Jin D, Xu J, Guo Y (2021) Structure features, selenylation modification, and improved anti-tumor activity of a polysaccharide from Eriobotrya japonica. Carbohydr Polym 273(1):118496
12. Chen Y, Lian Y, Chao J (2021) Protective effect of Lycium barbarum polysaccharides and capsaicin in rats with dextran sulfate sodium-induced ulcerative colitis via anti-inflammation and antioxidation. Curr Dev Nutr 5(Supplement2):306

13. Zhou M, Zheng X, Ha Z, Le Li, Zhang L, Liu M, Liu Z, Peng M, Wang C, Qin Li, Li D (2021) Effect of Lactobacillus plantarum enriched with organic/ inorganic selenium on the quality and microbial communities of fermented pickles. Food Chem 365:130495

14. Huang S, Yang W, Huang G (2020) Preparation and activities of selenium polysaccharide from plant such as Grifola frondosa. Carbohydr Polym 242:116409

15. Gu Y, Qiu Y, Wei X, Li Z, Hu Z, Gu Y, Zhao Y, Wang Y, Yue T, Yuan Y (2020) Characterization of selenium-containing polysaccharides isolated from selenium-enriched tea and its bioactivities. Food Chem 316:126371

16. Dong F, Zheng H, Jeong W, Chung S, Qu Z, Zou X, Liu C, Xiang Q, Feng $F$ (2021) Synthesis, characterization, and antioxidant activity in vitro of selenium-Euryale ferox Salisb. polysaccharide. Appl Biol Chem 64(1):59-61

17. Wang X, Yuan L, Bao Z, Fu B, Jiang P, Ma T, Lin S (2021) Screening of uric acid-lowering active components of corn silk polysaccharide and its targeted improvement on renal excretory dysfunction in hyperuricemia mice. J Funct Foods 86:104698

18. MaY WX, Gao S, Zhang J (2016) Optimized enzyme-assisted microwave extraction and potential inhibitory action against a-glucosidase of polysaccharides from sweet corncobs. Phyton-Int J Exp Bot 85:61-65

19. Palmu S, Kuneinen S, Kautiainen H, Eriksson J, Korhonen P (2021) Body surface area may explain sex differences in findings from the oral glucose tolerance test among subjects with normal glucose tolerance. Nutrition $31(9): 2678-2684$

20. Jin PJ, Young LW (2021) Anti-glycation effect of Ecklonia cava polysaccharides extracted by combined ultrasound and enzyme-assisted extraction. Int J Biol Macromol 180:684-691

21. Wang Z, Dai B, Zhang $L$ (2016) Optimization of technological parameters and structural analysis of preparation of polysaccharide selenate from pomegranate rind. Food Sci 37(10):40

22. Shang L, Wu S, Zhang C (2016) Preparation characterization and activity analysis of selenium polysaccharide from pumpkin. Food Sci 37(19):48

23. Spectrochimica Acta Part A (1993) Spectrochimica Acta Part A: Molecular Spectroscopy 49(9):1433

24. Corsaro M, Castro C, Naldi T, Parrilli M, Tomás J, Regué M (2005) ${ }^{1} \mathrm{H}$ and ${ }^{13} \mathrm{C}$ NMR characterization and secondary structure of the $\mathrm{K} 2$ polysaccharide of Klebsiella pneumoniae strain 52145. Carbohydr Res 340(13):2212-2217

25. Habibi Y, Heyraud A, Mahrouz M, Vignon M (2004) Structural features of pectic polysaccharides from the skin of Opuntia ficus-indica prickly pear fruits. Carbohyd Res 339:1119-1127

26. Mukhiddinov Z, Khalikov D, Abdusamiev F, Avloev C (2000) Isolation and structural characterization of a pectin homo and ramnogalacturonan. Talanta 53:171-176

27. Perrone P, Hewage C, Thomson A, Bailey K, Sadler I, Fry S (2002) Patterns of methyl and O-acetyl esterification in spinach pectins: new complexity. Phytoc hemistry 60:67-77

28. Renard C, Jarvis M (1999) Acetylation and methylation of homogalacturonans 1: optimisation of the reaction and characterisation of the products. Carbohyd Polym 39:201-207

29. Wei Y, Zhao Q, Wu Q (2019) Efficient synthesis of polysaccharide with high selenium content mediated by imidazole-based acidic ionic liquids. Carbohyd Polym 203:157-166

\section{Publisher's Note}

Springer Nature remains neutral with regard to jurisdictional claims in published maps and institutional affiliations. 